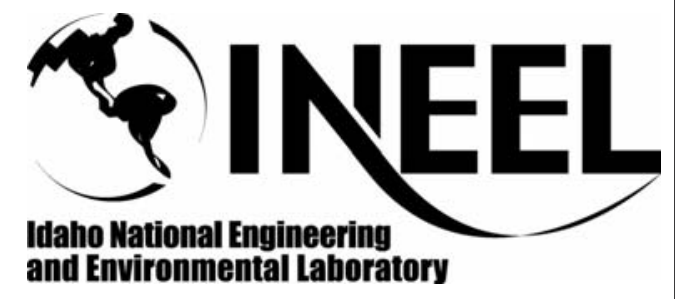

and Environmental Lahoratory
INEEL/CON-01-00650

PREPRINT

\section{Imaging Laser \\ Ultrasonics}

\section{Measurement of the \\ Elastodynamic \\ Properties of Paper}

\author{
Ken L. Telschow \\ Vance A. Deason
}

\section{October 2001}

\section{IEEE International Ultrasonic Symposium}

This is a preprint of a paper intended for publication in a journal or proceedings. Since changes may be made before publication, this preprint should not be cited or reproduced without permission of the author.

This document was prepared as an account of work sponsored by an agency of the United States Government. Neither the United States Government nor any agency thereof, or any of their employees, makes any warranty, expressed or implied, or assumes any legal liability or responsibility for any third party's use, or the results of such use, of any information, apparatus, product or process disclosed in this report, or represents that its use by such third party would not infringe privately owned rights. The views expressed in this paper are not necessarily those of the U.S. Government or the sponsoring agency. 


\title{
Imaging Laser Ultrasonics Measurement of the Elastodynamic Properties of Paper
}

\author{
Ken L. Telschow* and Vance A. Deason \\ Idaho National Engineering and Environmental Laboratory \\ Idaho Falls, ID 83415-2209, USA
}

Abstract - Many sheet and plate material industries
(e.g. paper) desire knowledge of the anisotropic
stiffness properties of their material to optimize the
manufacturing process. A determination of the
anisotropic elastic matrix would be very beneficial for
determination of parameters, such as microstructura
texture, fiber or grain orientation and stiffness. The
propagation of ultrasonic waves in plates is a method
for determining the anisotropic elastic properties in
nondestructive manner. Laser ultrasonics offers
noncontacting means to implement these measurements in the workplace by employing pulsed or modulated light to excite symmetric and antisymmetric plate waves concurrent with optical interferometric detection. Measurements can then be performed along the machine and cross directions to obtain parameters that are used empirically for process monitoring.

Recently, the INEEL has developed a full-field view laser based ultrasonic imaging method that allows simultaneous measurement of plate wave motion in all planar directions within a single image without scanning. The imaging measurements are based on dynamic holography using photorefractive materials for interferometric detection and are operated at normal video rates. Results from this laser based imaging approach are presented that record Lamb wave mode wavefronts in all planar directions from localized sources in a single image. Specific numerical predictions for flexural wave propagation in distinctly different types of paper accounting fully for orthotropic anisotropy are presented and compared with direct imaging measurements. Very good agreement with theoretical calculations is obtained for the lowest antisymmetric plate mode in all planar directions using paper properties independently determined by others.

\section{INTRODUCTION}

Laser ultrasonics is now a well established approach for elastic wave measurements that does not require the transducers to be in physical contact with the sample. $[1,2,3]$ It is a technique that employs lasers to both generate and detect elastic wave motion at the surface of materials. Typically, a pulsed laser delivers a high intensity but very short impulse to the sample through absorption of light at the surface. Operation in the thermoelastic regime is nondamaging and well understood but usually much less efficient than the ablation regime. An optical interferometer serves for detection by demodulating the small phase modulation that the elastic wave motion produces on a continuous probe laser beam. $[4,5]$ The laser ultrasonic technique has been used to measure elastic properties of thin plates $[6,7,8]$ and paper. $[9,10,11]$ Laser ultrasonics offers great potential for implementation in the field and even in situ during the paper making process. $[12,13]$

Paper is inherently anisotropic due to the deliberate aligning of the fibers to provide large strength in the pulling or machine direction (MD). Figure 1 shows the elastic wave speeds as a function of frequency for a paper similar to that used in copy machines. Orthotropic symmetry is assumed and elastic constants used that were determined through contact ultrasonic testing of specific modes. Only propagation along the principal axes is shown. Both antisymmetric $\left(\mathrm{A}_{0}\right)$ and symmetric $\left(\mathrm{S}_{0}\right)$ modes exhibit dispersion that can complicate the inversion of data from pulse-echo measurements for elastic constant determination. Because of this dispersion, common practice is to use the speed of the $S_{0}$ wave along both the principal directions to characterize the paper. This approach is typically limited to the MD and CD directions in practice, which may not actually be along the principal directions if there is any 
misalignment of the processing machine. In fact, this degree of misalignment is a needed measurement variable for improving process control.

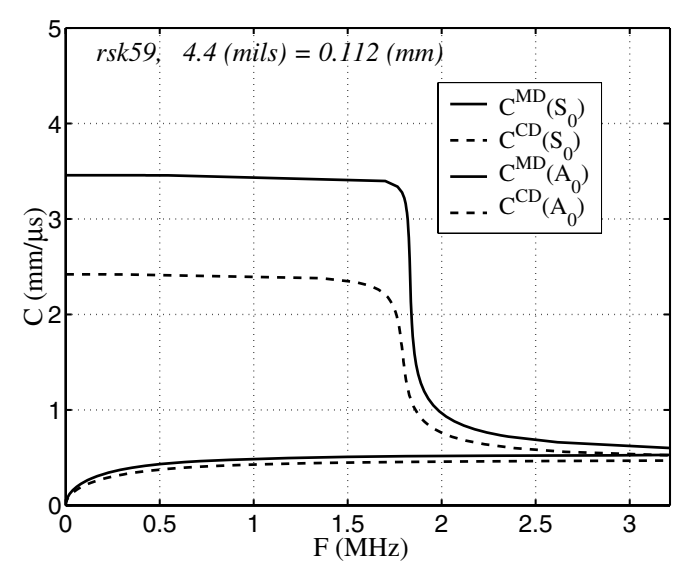

Figure 1. Plate wave dispersion curves for raw stock paper, similar to copy paper.

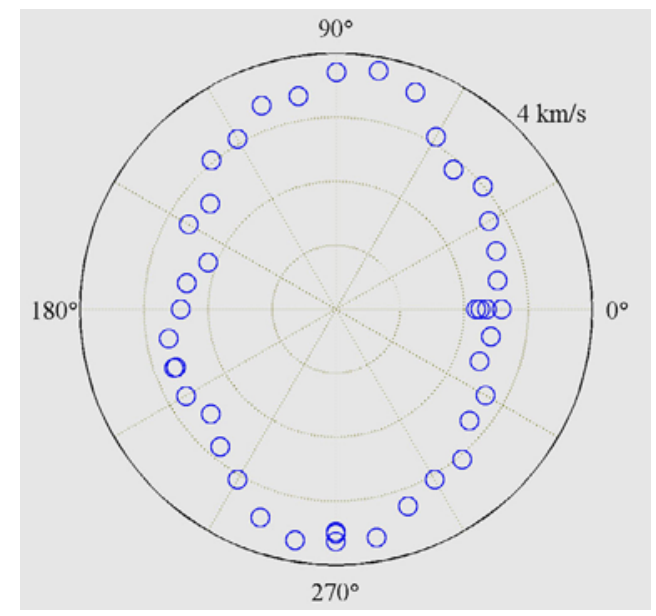

Figure 2. Wave speeds as a function of planar angle with respect to $\mathrm{CD}$ for raw stock paper made with single point laser ultrasonic methods.

Figure 2 shows the results of measurement of the $S_{0}$ wave speed as a function of planar angle made with the single point laser ultrasonic technique. The anisotropy is readily apparent producing wave speeds of $3.75 \mathrm{~km} / \mathrm{sec}(\mathrm{MD})$ and $2.53 \mathrm{Km} / \mathrm{sec}(\mathrm{CD})$ for a ratio of 1.48 that compares well with a value of 1.41 determined by the contact technique.

Currently, scanning of single point measurements over the paper surface is being compared to theoretical modeling of paper anisotropy restricted to principal directions. However, more information about the paper microstructure (such as texture orientation with respect to the drawing direction) is available through accounting for orthotropic anisotropy in all planar directions of the paper (i.e. along directions other than the elastic symmetry axes) and implementation of full field imaging of elastic wave propagation. The remainder of this paper presents a full-field imaging experimental approach that provides a means for accomplishing these goals.

\section{DYNAMIC HOLOGRAPHIC DETECTION}

A new approach to optical detection of ultrasonic motion in a microscopic geometry has been developed using photorefractive materials [14]. Twowave mixing of signal and reference beams within these materials can produce an output beam that demodulates the phase difference into an intensity variation. This method is similar to many interferometric techniques for phase demodulation of ultrasonic motion, except that active stabilization between the two beam paths at the phase quadrature point is not required. The photorefractive approach automatically produces a quadrature phase shift between signal and reference beams when the charge transport mechanism is diffusive [15]. Another advantage is that complicated optical wavefronts from surfaces with rough features are readily accommodated through the volume holographic process to produce the optimum reference beam for interferometric detection (i.e. samples with rough surfaces can be investigated with little loss of sensitivity due to speckle averaging). Finally, the ultimate goal of these measurements is to obtain images of ultrasonic motion over the entire surface within a single video frame, not requiring scanning over the surface. This has been accomplished at $\mathrm{MHz}$ frequencies on surfaces from 1"x 1" to 6"x 6" for both resonant and traveling wave motion $[16,17]$.

A schematic of the optical detection process is shown in Figure 3. A solid state single frequency laser at $532 \mathrm{~nm}$ produces both the signal and reference beams. After suitable beam expansion, the sample is illuminated with the signal beam. A picture of the sample surface is imaged inside a photorefractive material along with the reference beam. The two beams are combined in a Bismuth Silicon Oxide (BSO) photorefractive crystal, operated in the charge diffusion mode. The resultant output 
beam from the two-wave mixing is then measured with a CCD video camera for full-field imaging or with a photodiode detector and lock-in amplifier for single point measurement.

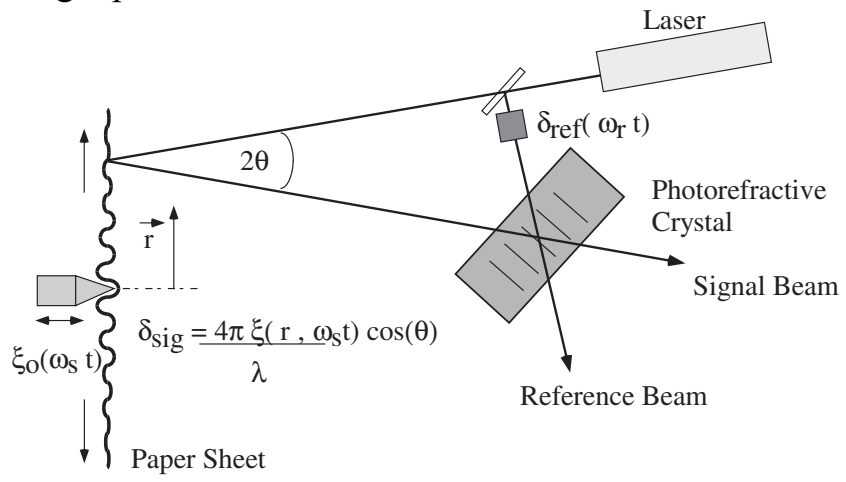

Figure 3: Photorefractive Optical Detection Schematic.

The local interference pattern inside the BSO forms regions of high and low intensity. Electrons are photoexcited in the bright regions and diffuse to the dark regions forming a volume distribution of charge separation that contains the phase difference information between the signal and reference images. The resultant charge distribution produces an electric field distribution known as the space charge field that locally alters the optical index of refraction through the electrooptic effect. An internal optical grating or more correctly a volume hologram forms within the BSO that diffracts energy from the reference beam exactly coaxial with the output signal beam. Subsequent detection at the CCD captures the intensity and phase information between the signal and reference images into a single image. The signal beam is modulated by the surface motion of the ultrasonic wave and an external electrooptic modulator is used to modulate the phase of the reference beam such that the difference frequency is at a value suitable for the optical detector (CCD or photodetector).

\section{OPERATION}

The out-of-plane motion of a vibrating surface produces a phase shift of an optical beam reflected normal to the surface of $\varphi_{S}(t)=\frac{4 \pi \xi}{\lambda}$, where $\xi(t)=\xi_{0} \cos (2 \pi f t+\psi)$ and $\lambda$ is the optical wavelength. Combining this beam with a reference beam phase modulated in a similar manner at the offset frequency $f+\Delta f$, produces an interference pattern with a narrowband component varying at the frequency $\Delta f$. Two-wave mixing within the photorefractive material produces an output beam with intensity of this component [18] of:

$$
\left|I_{A C}\right| \propto J_{0}\left(\varphi_{S}\right) J_{1}\left(\varphi_{S}\right) J_{0}\left(\varphi_{R}\right) J_{1}\left(\varphi_{R}\right)
$$

with $\varphi_{S}, \varphi_{R}$ the optical phase modulation depth on the signal and reference beams. It is apparent that both the signal and reference beam modulations produce an output intensity proportional to the product of the $0^{\text {th }}$ and $1^{\text {st }}$ order Bessel functions.

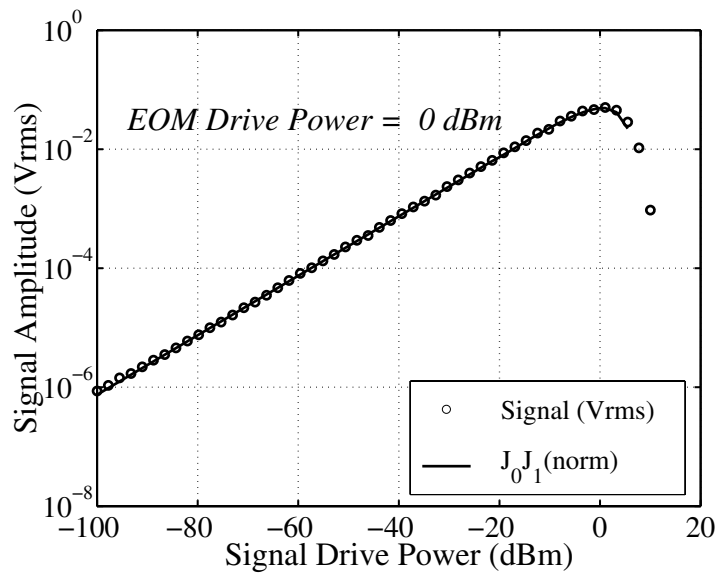

Figure 4: Calibration Phase Measurement showing agreement with equation 1 over five orders of magnitude.

This particular Bessel function product has a maximum value for a modulation depth of $\delta=1.08$ radians and is proportional to $\delta$ for smaller modulations. At an optical wavelength of $532 \mathrm{~nm}$, this maximum value corresponds to an ultrasonic displacement of $45.8 \mathrm{~nm}$. Figure 4 shows the results of measuring this response by driving the electrooptic modulator (EOM) as a function of amplitude at fixed frequency. The reference side is then set at that drive level for maximum phase modulation depth. Through use of a wide band DC-100 MHz EOM, operation at any ultrasonic frequency within this range can be performed. Signal beam modulations brought about 
by normal ultrasonic displacements are usually in the subnanometer to nanometer range. Figure 4 allows for easy absolute calibration of the detection sensitivity compared to the wavelength of light.

In operation, the difference frequency is set to somewhere between $1-30 \mathrm{~Hz}$, so that the video camera can track the image changes brought about by the moving grating. One then observes the ultrasonic wavefront traversing the screen at this difference frequency for traveling waves or the subsequent eigenmode wavefunctions for a resonant vibration. A single frame of this image is shown in figure $5 \mathrm{a}$.
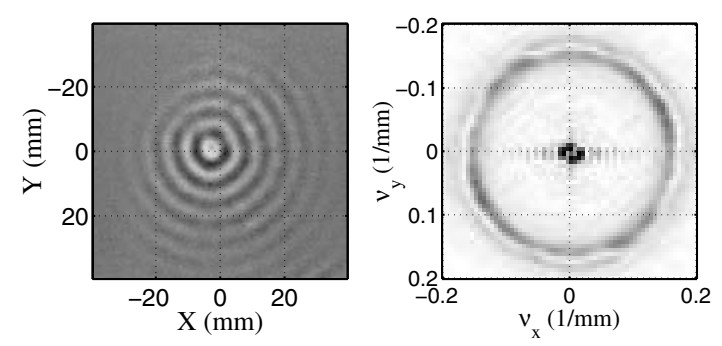

Figure 5. (a) Single frame image of $30 \mathrm{kHz}$ flexural waves emanating from a central point contact piezoelectric transducer in a $0.125 \mathrm{~mm}$ thick nickel sheet and (b) the Fourier transform of this image showing the wavenumber for this wave.

Any single frame has information on the wave propagation in all planar directions. This is most easily seen in the Fourier transform of the image, which produces a plot of the inverse wavelength (wavenumber) in all directions as shown in figure $5 b$. The magnitude of the Fourier transform of the traveling wave displacement shows real poles at the wavenumber for the traveling wave and imaginary poles of the same magnitude that contribute to satisfy the boundary conditions. Therefore, the Fourier transform image of the traveling wave displacement image is a single ring at the wavenumber delineating the propagating mode. Since, the nickel sheet was essentially isotropic, circular patterns are seen for both the wavefronts and the wavenumber images. Measurements at different frequencies allow for easy comparison with the known properties of nickel over a large frequency range, see Figure 6.

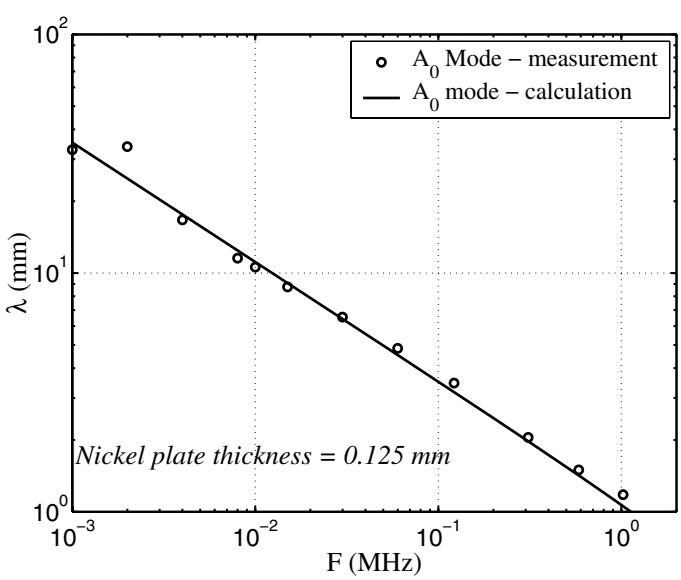

Figure 6. Calculation and measurement of the flexural mode wavelength for the isotropic nickel sheet over a wide frequency range.

\section{MEASUREMENTS IN PAPER}

Paper samples with elastic properties previously determined by contact piezoelectric methods were obtained form colleagues at the Institute of Paper Science and Technology (IPST) in Georgia. Figure 7 shows interesting wavefronts observed for the raw stock or copy paper. Measurements on paper were performed by driving a flexural wave from a point piezoelectric transducer, as before. The microstructure of paper and the elastic stiffness matrix are anisotropic as seen in the wavefront pattern and also in the transformed wavenumber image of Figure 8.

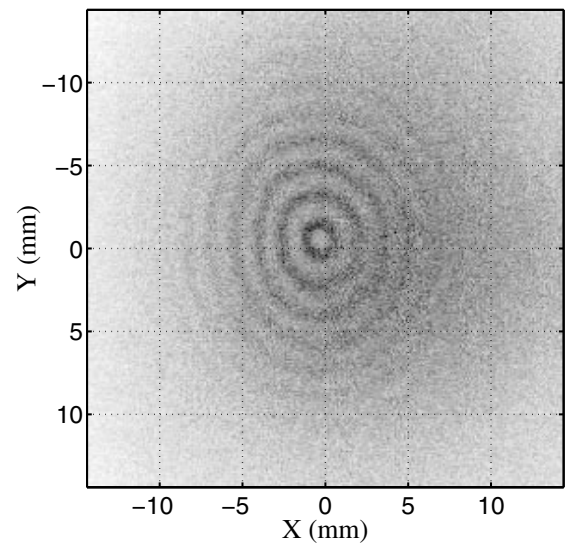

Figure 7. Single frame image of the lowest flexural mode in raw stock paper at $100 \mathrm{kHz}$. 


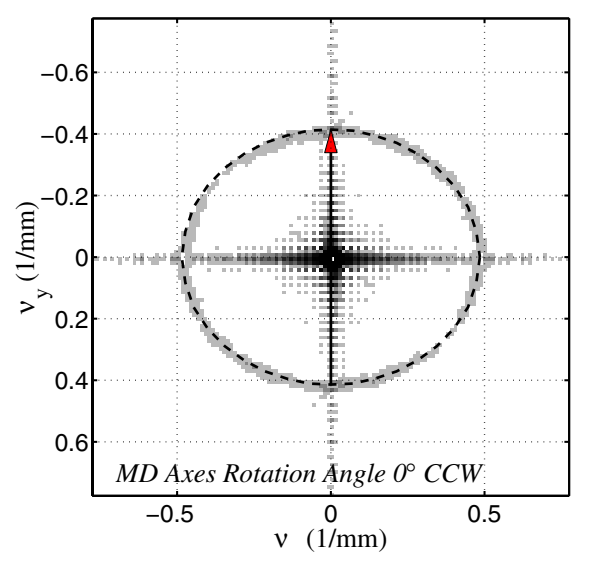

Figure 8. Transform image of the raw stock paper image showing the anisotropic wavenumber for this paper sample. The dashed line is the theoretical calculation assuming the MD axis is vertical.

Note that the ultrasonic wavefronts are clearly shown in all directions at once over $20 \times 20 \mathrm{~mm}$ region. Averaging of in phase and out of phase images was used to suppress the background surface image. The anisotropic nature of the ultrasonic wave propagation is clearly visible in the images as well as laser speckle.

Fourier transform of the displacement image results in an image of the "wavenumber" diagram for this material; that is, an image showing $(1 / \lambda)$ in all directions, where $\lambda$ is the ultrasonic $\mathrm{A}_{0}$ mode wavelength at the measurement frequency. Processing the spatial image in this manner is particularly convenient as it utilizes all the data the image provides. Even images with much reduced signal to noise ratio still result in useable images for comparison to the theoretical predictions. Superimposed onto the FFT image of Figure 8 is the prediction resulting from an exact theoretical calculation that is valid for all planar directions in an orthotropic sheet performed by Professor Subhendu Datta's group at the University of Colorado. There are no adjustable parameters in this comparison. The theoretical predictions are based entirely on the elastic coefficients, density and thickness parameters obtained from independent IPST measurements. The exceptional agreement between the theoretical predictions and the experimental data from the camera measurements is readily apparent and testifies to the overall accuracy of the entire theoretical and experimental process followed. [19]

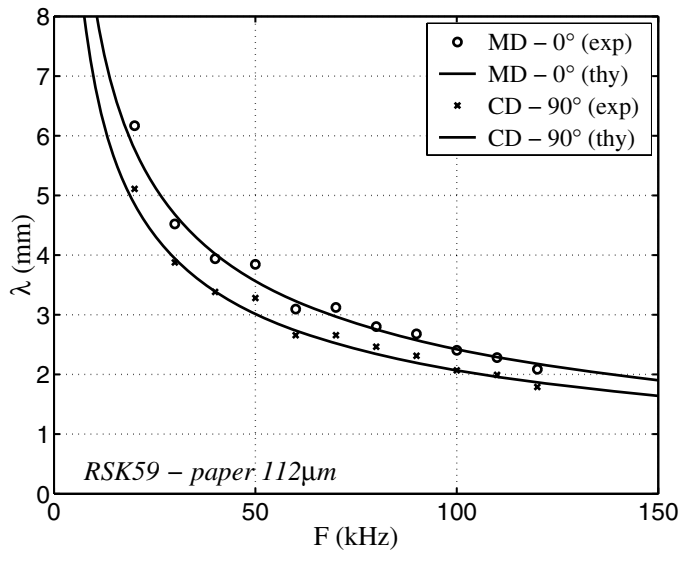

Figure 9. Measured and predicted wavelengths for the antisymmetric flexural mode in raw stock $0.112 \mathrm{~mm}$ thick paper.

Decrease of the ultrasonic wavelength with frequency for the antisymmetric mode is also clearly observed and determined from the images. Figure 9 shows the wavelengths measured for the $A_{0}$ mode of the raw stock paper as a function of frequency along with predictions described above for the principal axes.

A significant advantage of the ultrasonic wave fullfield measurement method is the fact that data in all planar propagation directions is obtained simultaneously in one image. This allows one to determine the elastic stiffness in both the MD and CD directions as well as obtain a measure of the alignment of the paper axes with the manufacturing direction. A misalignment produces a rotation of the image, as can be seen from figures $10(a)(b)(c)$ for the raw stock paper. The figure shows the data along with the theoretical calculation plotted for three different rotation angles between them with respect to the MD direction. The predicted wavenumber image is rotated at $-10,+8,+20$ degrees from the vertical in a counter clockwise $(\mathrm{CCW})$ direction. A best fit between the prediction and the data occurs for a rotation of +8 degrees $\mathrm{CCW}$ as measured by a cross correlation between the image and the prediction. Therefore, we can conclude that the actual orientation of the elastic anisotropy (due mostly to the paper fiber alignment) is rotated by this amount with respect to the vertical or MD direction, that is referenced from the cut paper edges. This information would be difficult to obtain from single point measurements, unless a large scanned set of measurements were performed 
(a)

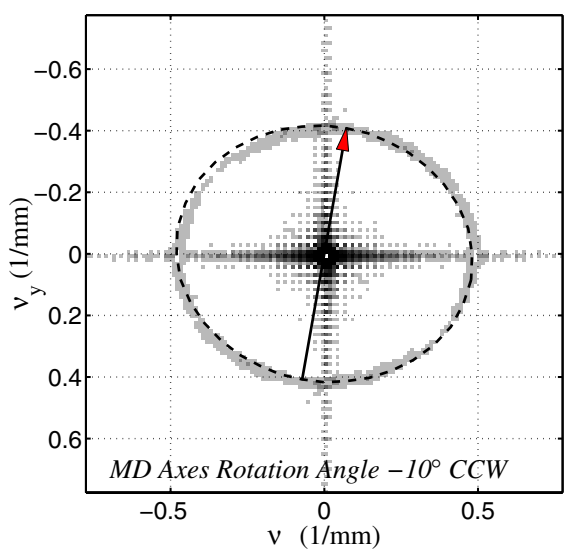

(b)

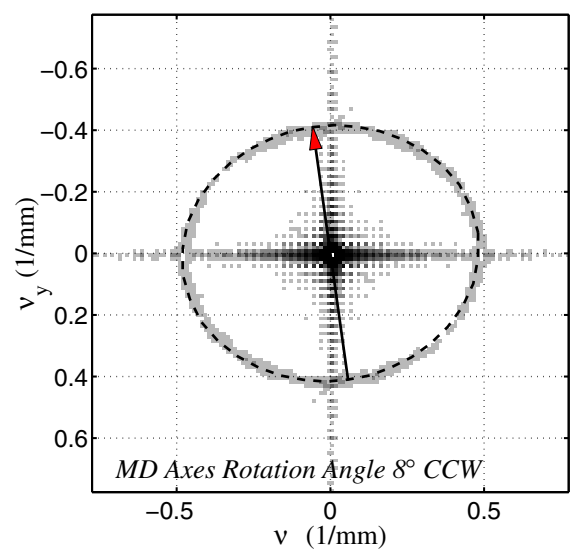

(c)

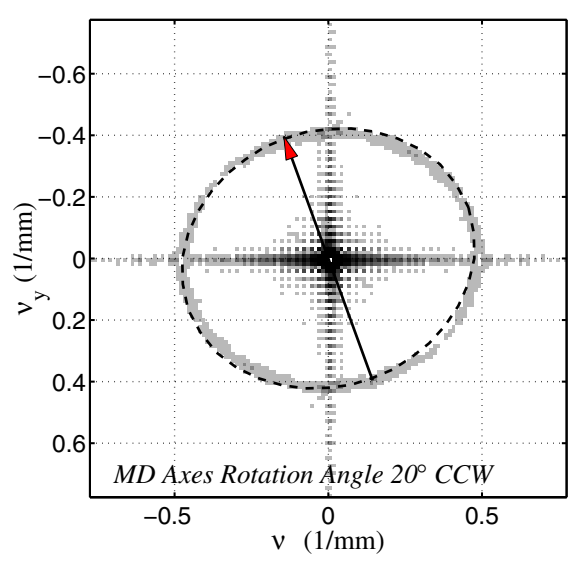

Figure 10. Comparison of predicted wavenumber for RSK59 paper oriented at various rotation angles of (a) -10 , (b) 8 (best fit), (c) 20 degrees $\mathrm{CCW}$ with respect to the MD (vertical) axis.

\section{MICROSTRUCTURAL TEXTURE AND STRESS}

Microstructural texture and residual or applied stress are well known to alter the stiffness of plates. In particular, alignment of elongated grains due to work hardening produces a texture in the microstructure that shows up as an anisotropy in the elastic coefficients. Figure 11 shows results from a sheet of Brass in the "as produced" state. The manufacturing process produces significant texture in the microstructure that shows up as anisotropy in the elastic stiffness. The top figure shows the antisymmetric wavefront at $33 \mathrm{kHz}$ for an "as produced" $0.061 \mathrm{~mm}$ thick brass sheet. The data

(a)
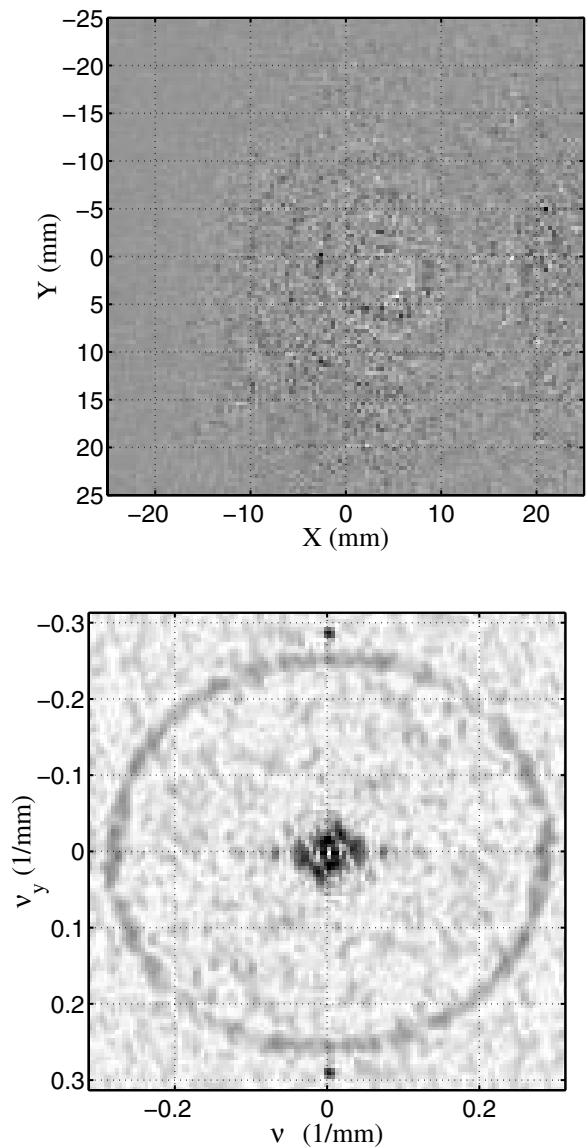

(b)

Figure 11. Spatial image (a) and wavenumber (transform) (b) of a flexural wave in $0.061 \mathrm{~mm}$ Brass sheet "as produced" showing the anisotropy brought about by microstructural texture under no applied stress. 


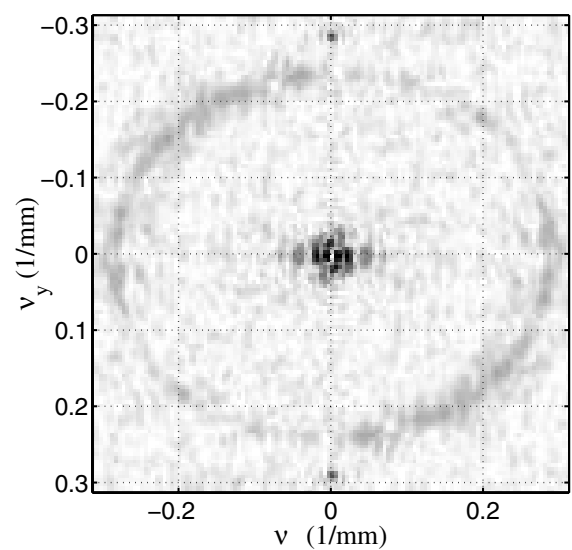

(a)

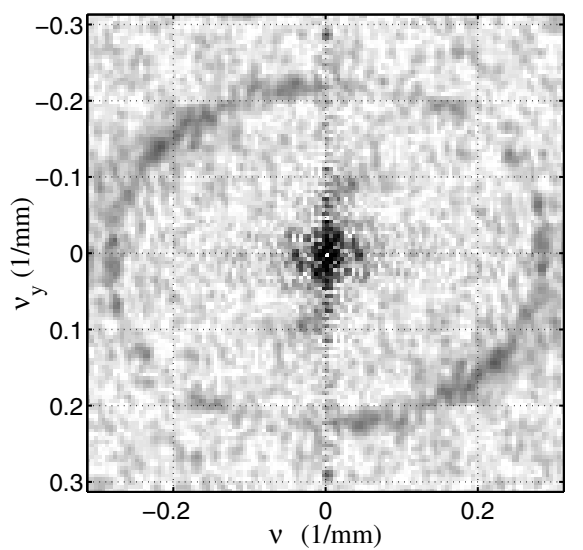

(b)

Figure 12. Wavenumber images for the Brass sheet of Figure 11 with (a) $47 \mathrm{MPa}$ and (b) $94 \mathrm{MPa}$ of stress applied along the vertical direction.

quality is less than optimal, as can be seen. The second part of this figure shows the Fourier transform image, where the anisotropic elastic stiffness can be seen even for the rather poor signal to noise of the original image data. Signal averaging and subtracting correlated in-phase and 180 degree outof-phase images improves the data when the elastic wave displacement amplitude is near the minimum detectable value. Flexural wave speeds increase with internal tensile stress whether brought about by residual or applied conditions. Application of stress along the vertical direction subsequently increases the plate wave speed in that direction and shows increased narrowing of the transform wavenumber image in a manner consistent with predictions using a partial wave method of calculating the wave speed in the presence of uniaxial stress. Transforms of the original image data are presented in Figure 12 for the addition of uniaxial stress.

\section{ADDITIONAL USES FOR THE LASER ULTRASONIC CAMERA}

The laser ultrasonic camera can be used for other imaging measurements such as recording ultrasonic waves scattering from defects inside of materials. [20] A recent test of this modality was performed by imaging scattered radiation from mock defect (drilled flat bottom hole) with a longitudinal transducer placed on the outside of an aluminum block. Figure 13 shows the arrangement as well as the images recorded both at the top and bottom of the block. Clearly, the images show much about the scattering and propagation process for longitudinal waves in the aluminum block. In particular, the top surface shows diffraction from the hole, as if from a round aperture, as well as a wavefront pattern from the plane waves impinging the surface from an oblique angle with respect to the normal. For comparison, the bottom image shows diffraction effects from the defect in the near field of the hole, circular region in the center.

\section{CONCLUSIONS}

A new imaging ultrasound modality, a laser ultrasonic camera employing photorefractive dynamic holography, has been described that is capable of investigating the elastic behavior of isotropic and anisotropic materials in several ways. The camera allows one to observe ultrasonic motion in real-time at video frame rates using heterodyning of the motion to slower speeds. The method is capable of flat frequency response over a wide range and is fully functional on samples with rough surfaces. The method has been proven over a large frequency range from $\mathrm{Hz}$ to $\mathrm{MHz}$ with a sensitivity of $0.1 \mathrm{~nm}$ of absolute surface motion. Subsequent processing of the images in the Fourier transform domain yields determination of elastic anisotropy through wavenumber images that reflects a material's microstructural symmetry, texture and residual or applied stress. Many other acoustical imaging modes can be implemented with this camera and an example of imaging scattered waves from a buried flaw in an aluminum block was presented. 

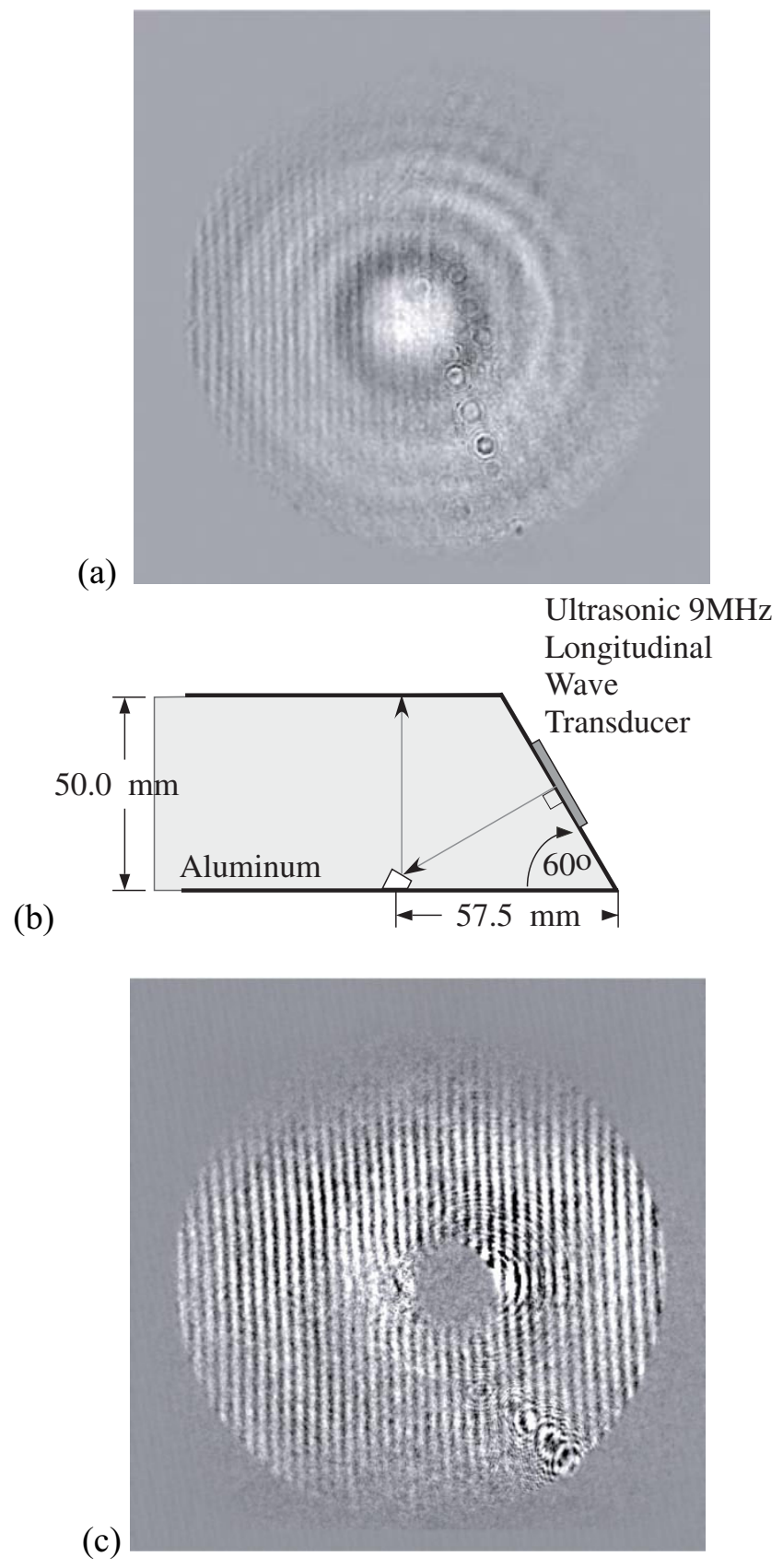

Figure 13. Image at the top surface (a) of an aluminum block (b) from ultrasonic waves scattered from an internal flat bottom hole, the image at the bottom surface is shown in (c).

\section{ACKNOWLEDGEMENTS}

The authors thank S. M. Watson and R. S. Schley for experimental help, Professor Subhendu Datta and O. Mukdadi of the University of Colorado for the modeling calculations, and Dr. J. Gerhartstein, Dr. P. Brodeur and Dr. C. Habeger of the Institute of Paper Science and Technology, Atlanta, GA, for the elastic constant matrices for the paper samples. This work was sponsored by the U.S. Department of Energy, Office of Energy Research, Office of Basic Energy Sciences, Engineering Research and the INEEL Laboratory Directed Research \& Development program under DOE Idaho Operations Office Contract DE-AC07-99ID13727.

\section{REFERENCES}

[1] C. B. Scruby, R. J. Dewhurst, D. A. Hutchins and S. B. Palmer, "Laser Generation of Ultrasound in Metals," Research Techniques in Nondestructive Testing, vol.Ê2, R. S. Sharp editor (Academic press, N.Y., 1982), 281-327.

[2] F. V. Bunkin, A. A. Kolomensky and V. G. Mikhalevich, Lasers in Acoustics, (Harwood Publisher Academic GmbH, Chur, Switzerland, 1991)

[3] V. E. Gusev and A. A. Karabutov, Laser Optoacoustics, (A. I. P. New York, 1993)

[4] J.-P. Monchalin, "Optical Detection of Ultrasound," IEEE Trans. on UFFC $\underline{33}$, 485-490, (1986).

[5] J. W. Wagner, "Optical Detection of Ultrasound," Physical Acoustics, Vol. XIX, Eds. Thurston, R. N., and Pierce, A.D., (Academic Press, NY, 1990) Chp. 5.

[6] R. J. Dewhurst, C. Edwards, A. D. W. McKie, S.B. Palmer, "Estimation of the thickness of thin metal sheet using laser generated ultrasound", Appl. Phys. Letters 51(14), 10661068, (1987).

[7] D. A. Hutchins and K. Lundgren, "A laser study of transient Lamb wave in thin materials", J. Acoust. Soc. Am. 85(4), 1441-1448, (1989). 
[8] H. Nakano and S. Nagai, "Laser generation of antisymmetric Lamb waves in thin plates", Ultrasonics 29, 230-234, (1991).

[9] M. A. Johnson, Y. H. Berthelot, P. H. Brodeur, and L. A. Jacobs, "Investigation of laser generation of Lamb waves in copy paper", Ultrasonics 34, 703-710, (1996).

[10] J. C. Cheng and Y. H. Berthelot, "Theory of laser-generated transient Lamb waves in orthotropic plates", J. Phys. D. 29, 1857-1867, (1996).

[11] P. H. Brodeur, M. A. Johnson, Y. H. Berthelot, and J. P. Gerhardstein, "Noncontact laser generation of Lamb waves in paper", J. Pulp and Paper Sci. 23(5), J238-J242, (1997).

[12] P.L. Ridgway *, A.J. Hunt, M. Quinby-Hunt, R.E. Russo, "Laser ultrasonics on moving paper," Ultrasonics 37 ( 1999) 395-403.

[13] J. B. Walter, K.L. Telschow, J.P. Gerhardstein, B.M. Pufahl, E.M. Lafond, and P.H. Brodeur, "Fabry-Perot Laser Ultrasonic Elastic Anisotropy Measurements in Paper on a Moving Web," CP509, Reviews of Progress in Quantitative $N D E$, edited by D. O. Thompson and D. E. Chimenti (American Institute of Physics, 1-56396-930-0), 247-254, (2000).

[14] P. Yeh, Introduction to Photorefractive Nonlinear Optics, (John Wiley, New York, 1993).

[15] R. K. Ing and J. P. Monchalin, "Broadband optical detection of ultrasound by two-wave mixing in a photorefractive crystal," Appl. Phys. Lett. 59, 3,233 (1991).

[16] T. C. Hale, K. L. Telschow, and V. A. Deason, "Photorefractive optical lock-in vibration spectral measurement," Applied Optics, 111, $8,248-8,258$ (1997).

[17] K. L. Telschow, V. A. Deason, R. S. Schley and S. M. Watson, "Direct Imaging of Lamb Waves in Plates using Photorefractive Dynamic Holography," J.Acoust. Soc. Am. 106(5), 25782587 (1999).

[18] K. L. Telschow, V. A. Deason, K. L. Ricks and R. S. Schley, "Photorefractive laser ultrasound spectroscopy for materials characterization," Nondestructive Characterization of Materials VIII, ed. R. E. Green, Jr., (Plenum Press, N Y, 1998) 79-84.
[19] O. Mukdadi, S. K. Datta, K. L. Telschow and V. A. Deason, "Ultrasonic Guided Waves in Thin Orthotropic Layers: Theoretical Analysis and Dynamic Holographic Imaging Measurement," accepted for publication in the journal IEEE Transactions: Ultrasonics, Ferroelectrics and Frequency Control (2001).

[20] V. A. Deason, K. L. Telschow, S. M. Watson, "Ultrasonic imaging of subsurface objects using photorefractive dynamic holography," Proceedings of the 46th Annual Meeting of the SPIE, San Diego, CA, July 29, 2001 (paper 4448-22).

* Ken Telschow: telsch@inel.gov 